\title{
Binary Liquid Mixtures Form Flattened Droplets
}

\section{Droplets made of a mixture of two liquids with different volatilities take on a pancake shape.}

\section{By Sophia Chen}

$\mathrm{R}$

esearchers seek to control the motion and behavior of small droplets for various applications, ranging from automating chemical and biological experiments to fabricating devices such as liquid lenses. Typically, models assume that the droplets stay spherical, even as they evaporate. Now, Amir Pahlavan of Princeton University and colleagues have shown that, under certain conditions, some droplets can flatten into pancake-like shapes. The team says that they aim to use their finding to engineer droplets that can toggle between shapes.

The researchers conducted experiments with picoliter-volume droplets that contained a mixture of two different alcohols, isopropanol and butanol. They deposited each droplet on a glass surface and then monitored it with a microscope.

Within a tenth of a second of deposition, Pahlavan and colleagues observed that droplets containing more than $40 \%$ isopropanol morphed from initially spherical to flat to spherical again. They explained the behavior as follows: While the liquids

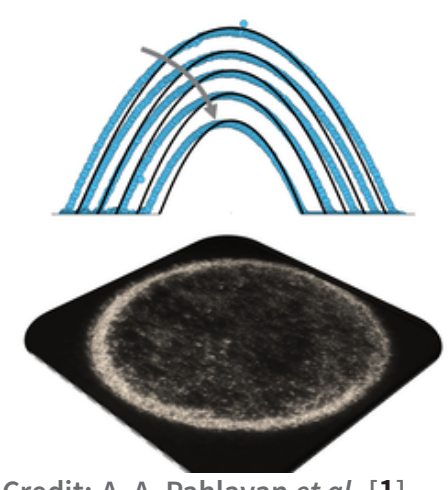

Credit: A. A. Pahlavan et al. [1]

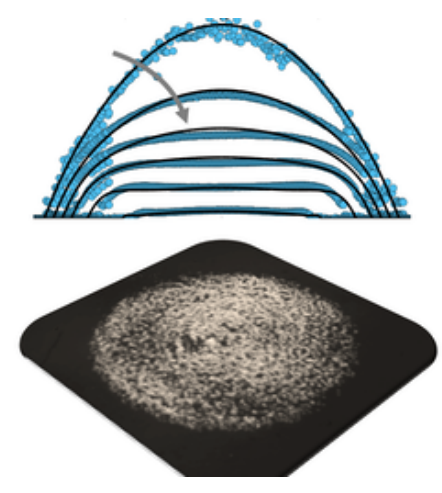

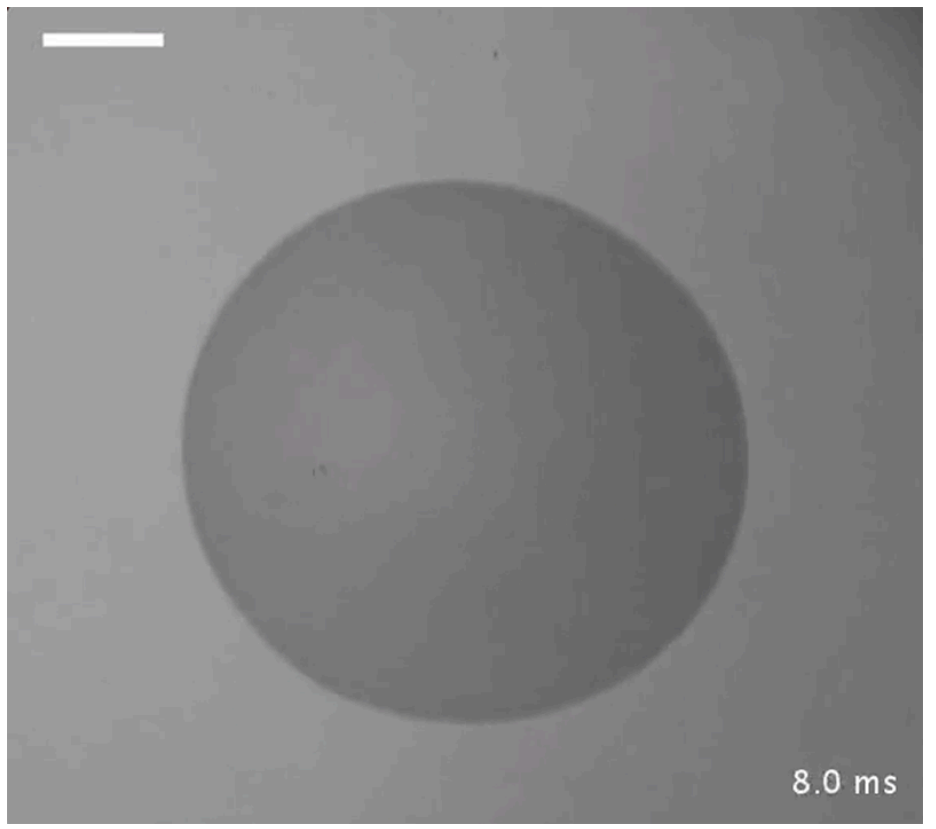

Video 1: Birds-eye view of the evaporation of a droplet containing $80 \%$ isopropanol. The team used the light and dark stripes to reconstruct the drop's height profile.

Credit: A. A. Pahlavan et al. [1]

in each droplet were initially well-mixed, the isopropanol evaporated much more quickly than the butanol, creating heterogeneities in the flows within the fluid, which deformed the drop.

In more detail, simulations performed by the team show that the shape changes were due to two competing flows: capillary flow, which arises from pressure variations within the drop, and Marangoni flow, which arises from a surface tension gradient 
along the droplet interface. The Marangoni flow caused liquid within the droplet to flow from the droplet's center to its outer edge; the capillary flow opposed that motion. When the Marangoni flow dominated, it created the pancake shape. Over time, the capillary flow overtook the Marangoni flow, and the droplets again became spherical. The results suggest that adding a more volatile liquid to a droplet could provide a way to tune a droplet's shape.
Sophia Chen is a freelance science writer based in Columbus, Ohio.

\section{REFERENCES}

1. A. A. Pahlavan et al., "Evaporation of binary-mixture liquid droplets: The formation of picoliter pancakelike shapes," Phys. Rev. Lett. 127, 024501 (2021). 